\title{
First report of alfalfa mosaic virus in Cayratia japonica, Justicia procumbens and Veronica persica in China
}

\author{
Shi Qi Gao ${ }^{1} \cdot$ Jing Liang ${ }^{1} \cdot$ Kai Guo $^{1} \cdot$ Xiang Zhou $^{1} \cdot \mathrm{Xiu} \mathrm{Su}^{1}$ (1) \\ Received: 4 April 2019 / Accepted: 3 September 2019 / Published online: 12 September 2019 \\ (C) Società Italiana di Patologia Vegetale (S.I.Pa.V.) 2019
}

Keywords Alfalfa mosaic virus · Deep sequencing · Cayratia japonica (Thunb) Gagnep · Justicia procumbens L. · Veronica persica Poir

Alfalfa mosaic virus (AMV), first discovered from alfalfa (Medicago sativa) in America (Weimer 1931) has a wide host range including many important economic crops in the Fabaceae and Solanaceae (Fletcher 2001). The widespread weed plants of Cayratia japonica (Thunb.) Gagnep, Justicia procumbens L. and Veronica persica Poir. were observed showing bright yellow or mild leaf mosaic symptoms in fields of Hangzhou City, Zhejiang Province, China in 2013. Symptomatic leaf samples of each weed species were collected, and tested for viral infection by deep sequencing of small RNA (Su et al. 2016). High-quality sequence reads were assembled to contigs which were specifically assigned to AMV by BLASTN search. To further confirm AMV infections, reverse transcription (RT)-PCR was performed on five symptomatic leaf samples and one symptomless sample for each weed, using the specific primer pair based on contigs sequences (amv-cp-f1, 5'- GCTGCTTTACGCAAAGCTCA AC-3'; amv-cp-r1, 5'-GGAGCGAATAGGACTTCATACC$\left.3^{\prime}\right)$. PCR products of the expected size ( $690 \mathrm{bp}$ ) were obtained from all the symptomatic samples, but not the symptomless samples. The sequenced amplicons from $C$. japonica (KX377691), J. procumbens (KX377692), and V. persica (KX377693) showed the highest nt sequence identity of 99\% with AMV isolate TBN from Iran (KM655878.1).

Xiang Zhou

xzhou@zafu.edu.cn

Xiu Su

suxiu@zafu.edu.cn

1 National Joint Local Engineering Laboratory of Biopesticide high-efficient Preparation, State Key Laboratory of Subtropical Silviculture, School of Forestry and Biotechnology, Zhejiang A\&F University, Hangzhou 311300, China
Inoculation of extracts from these AMV-infected weed plants caused weak mosaic symptoms on Nicotiana benthamiana and Chenopodium amaranticolor at 10-15 days after inoculation, and all the inoculated plants tested positive for AMV by RT-PCR with amv-cp-fl/r1 primers. To our knowledge, this is the first report of C. japonica, J. procumbens, and $V$. persica as natural hosts of AMV. Considering the potential non-persistent aphid transmission as well as mechanical transmission, the AMV-infected hosts may serve as a natural reservoir and pose a risk to other ornamental plants and crops.

\section{References}

Fletcher JD (2001) New hosts of Alfalfa mosaic virus, Cucumber mosaic virus, Potato virus Y, Soybean dwarf virus, and Tomato spotted wilt virus in New Zealand. N Z J Crop Hortic 29(3):213-217

Su X, Fu S, Qian YJ, Zhang LQ, Xu Y, Zhou XP (2016) Discovery and small RNA profile of pecan mosaic-associated virus, a novel potyvirus of pecan trees. Sci Rep 6:26741

Weimer JL (1931) Alfalfa mosaic virus. Phytopathology 21:122

Publisher's note Springer Nature remains neutral with regard to jurisdictional claims in published maps and institutional affiliations. 